\title{
How do polydisperse repulsive colloids crystallize?
}

\author{
Robert Botet, ${ }^{* a}$ Bernard Cabane, ${ }^{b}$ Lucas Goehring, ${ }^{c}$ Joaquim Li ${ }^{\mathrm{c}}$ \\ and Franck Artzner ${ }^{d}$
}

Received 14th October 2015, Accepted 4th November 2015

DOI: $10.1039 / c 5 f d 00145 e$

A modified version of the Gibbs-ensemble Monte-Carlo method reveals how polydisperse charged colloidal particles can build complex colloidal crystals. It provides general rules that are applicable to this fractionated crystallization that stems from size segregation. It explains the spontaneous formation of complex crystals with very large unit-cells in suspensions of nanoparticles with a broad size distribution.

\section{Introduction}

Fundamental physics requires experiments to observe, and theory to explain. In principle, nothing more is needed for a complete understanding of phenomena. However, in some situations, this sharing of the work is not sufficient, either because the experimental data are counter-intuitive (disagreement with the theory) or because the theory itself is impracticable. If one of these cases arises, numerical simulations turn out to be the essential tool to complement our ideas through direct 'observation' of the theory or through artificial changes of the microscopic rules (as in a Maxwell's demon-like approach to analyze cause-toeffect mechanisms).

In the present article, we discuss results of numerical simulations dedicated to the comprehension of recent experiments in which a dispersion of polydisperse repulsive nanoparticles was compressed through osmosis. Details of the experiments will be published elsewhere. ${ }^{1}$ A suspension of ordinary colloidal silica particles (Ludox, polydispersity 14\%, average radius $8 \mathrm{~nm}$ ), was compressed through dialysis. This caused a spontaneous segregation of the particles, fractionated crystallization and multiple-phase coexistence (involving various crystal structures). For instance, the low-compression part of the phase sequence was seen to be a colloidal liquid at the lowest volume fractions, then nucleation of the

${ }^{a}$ Laboratoire de Physique des Solides, CNRS UMR8502, Univ. Paris-Sud, Université Paris-Saclay, 91405 Orsay Cedex, France. E-mail: robert.botet@u-psud.fr; Fax: +3316915 6086; Tel: +3316915 6925

${ }^{b} L C M D, C N R S$ UMR 8231, ESPCI, 10 rue Vauquelin, 75231 Paris Cedex 05, France

${ }^{c}$ Max Planck Institute for Dynamics and Self-Organization (MPIDS), 37077 Göttingen, Germany

${ }^{d}$ Institut de Physique, CNRS UMR 6626, Univ. Rennes, 35042 Rennes, France 
BCC crystalline phase occurred at a silica volume fraction $\phi>0.19$, and then there was coexistence of the $\mathrm{BCC}+$ Laves $\mathrm{AB}_{2}$ crystal phases for $\phi>0.22$. The complete phase diagram was found to be much richer than expected according to current theories.

It is important to note that we do not describe here ordinary fractionated crystallization caused by different solubilities of coexisting substances, but a new kind of fractionation that organizes populations of particles with different sizes, and makes the best use of available space. Without any theory at hand to explain the formation of such uncommon structures as the Laves $\mathrm{AB}_{2}$ crystals, ${ }^{2}$ we used the Gibbs-ensemble Monte-Carlo method $^{3}$ to understand the mechanisms used by the system to sort the various particle sizes and to put them in the correct places.

Actually, we encountered many unanswered questions from the experimental data. The numerical simulations helped us to answer some of them. We replicate hereafter this particular state of mind, dividing our comprehension process in a series of short questions and discussing the answers given by the numerical study. The reader interested in a quantitative comparison between the experimental data and the numerical simulations should refer to ref. 1.

To close this Introduction, let us give a warning which might be important when comparing experimental data with numerical simulations: the regular Monte-Carlo method does not consider real kinetic processes, but is an efficient tool to search for the equilibrium state using the physical ground of free energy minimization. Therefore, the results presented below are linked to the equilibrium state. If this is not the case (in experiments, in kinetic numerical simulations), other intermediate crystalline structures may appear for some time as unstable phases.

\section{The system under study}

We consider a dispersion of spherical hard particles. The surface of each particle is electrically charged with $0.4 e$ per $\mathrm{nm}^{2}$. The particle radius is denoted: $a$, and its value is Gaussian-distributed with the average value $\langle a\rangle=8 \mathrm{~nm}$ and standard deviation $\sigma$, truncated to the range: [ $4 \mathrm{~nm} ; 12 \mathrm{~nm}$ ] (that is: no radius is smaller than $4 \mathrm{~nm}$ or larger than $12 \mathrm{~nm}$ ). The polydispersity, $\delta$, of this population is defined as: $\delta^{2}=\left\langle a^{2}\right\rangle /\langle a\rangle^{2}-1$. Then, the value of $\delta$ coincides with $\sigma /\langle a\rangle$ for the small values of $\sigma$, and has the limiting value $1 / \sqrt{12} \simeq 29 \%$ for the infinite value of $\sigma$ (rectangular distribution). Throughout the article, we shall take the value $\sigma=$ 1.2 realizing the polydispersity $\delta=15 \%$ of the population.

If large polydispersity (say $\delta>25 \%$ ) has to be considered, the influence of moments of order larger than 2 should be studied, that is to investigate the various distributions - such as log-normal or Schulz distributions - with the same values of $\langle a\rangle$ and $\delta$. In related problems, the shape of the radius-distribution is known to be unessential. ${ }^{4}$

We consider here the DLVO theory, ${ }^{5}$ in which the interaction energy between two charged particles, labelled $i$ and $j$, is generally of the Yukawa (screenedCoulomb) form:

$$
U_{i j} / k_{\mathrm{B}} T=Z_{i} Z_{j} \frac{l_{\mathrm{B}}}{r_{i j}} \mathrm{e}^{-\kappa r_{i j}}, \quad \text { for } r_{i j} \geq a_{i}+a_{j}
$$


where $Z_{i}$ is the effective charge of the particle $i$, and $r_{i j}$ the distance between their centers. $l_{\mathrm{B}}=0.7 \mathrm{~nm}$ is the Bjerrum length, and $\kappa=1 / l_{\mathrm{D}}$ is the screening parameter, with $l_{\mathrm{D}}=3 \mathrm{~nm}$ the Debye screening length in our experimental conditions. The values of $l_{\mathrm{B}}$ and $l_{\mathrm{D}}$ are expected to be constant throughout the system.

Two definitions have to be stated:

- The energy at contact (i.e. using (1) for $r_{i j}=a_{i}+a_{j}$ ), traditionally written: $\beta \varepsilon$, is a central parameter for discussion. Its averaged value is $\beta \varepsilon=21.6$ for the present set of parameters.

- The potential energy per particle at equilibrium is here denoted generically: $E$. It is defined as the sum of the pair-potentials (1) between all the couples of particles forming the equilibrated system, divided by the total number of particles, after having minimized the system free-energy using the Monte-Carlo method.

At small or intermediate volume fractions - namely: $\phi \leq 0.41$ for $\delta=15 \%$ - the Wigner-Seitz radius is larger than $\langle a\rangle+l_{\mathrm{D}}$. In these conditions, the attractive van der Waals interaction between silica spheres in water is smaller than the thermal energy, $k_{\mathrm{B}} T$, and thus can be neglected. In the same way, we do not discuss here controversial additional weak long-ranged attractive pairwise potentials such as those appearing in the Sogami-Ise theory. ${ }^{6}$ Within our approach, such interactions would result in the same results as in the case of the purely repulsive DLVO potential, with an effective value of the osmotic pressure slightly larger than the experimental pressure. ${ }^{7}$

\section{Q1: which of the FCC or BCC crystalline structures should appear for a system made of a polydisperse distribution of repulsive particles at the thermodynamic equilibrium?}

A1: systems of monodisperse particles interacting through repulsive Yukawa potentials, form either FCC or BCC crystal structures in the equilibrium solid phase. $^{8}$ The BCC phase appears when $\phi<0.5$ and the Debye screening length $l_{\mathrm{D}}$ is larger than a threshold depending on $\phi$. In all other cases, the FCC phase is the solid structure at equilibrium.

In the case of polydisperse particles, the question is more complicated. Let us suppose first that the solid phase can only be FCC or BCC, without coexistence (note that, for the parameters as chosen, only the FCC crystalline phase can appear in the monodisperse case, since the value of $l_{\mathrm{D}} /\langle a\rangle \quad 0.38$ is below the liquid-BCC-FCC triple critical point). We performed random exchanges of couples of particles in the system composed of either a single FCC phase or a single BCC phase, according to the standard Metropolis Monte-Carlo algorithm, until thermodynamic equilibrium was reached. The evolution of the difference of energy at equilibrium per particle, $\left(E_{\mathrm{FCC}}-E_{\mathrm{BCC}}\right) / k_{\mathrm{B}} T$, versus the volume fraction is plotted in Fig. 1, and the monodisperse case is also plotted for comparison. Unlike the monodisperse case, the polydisperse population is seen to undergo a clear change in the stability of the BCC/FCC structure: when $\phi>0.3$, the BCC crystal is energetically more likely to appear than the FCC structure. Moreover this trend increases with the volume fraction. 


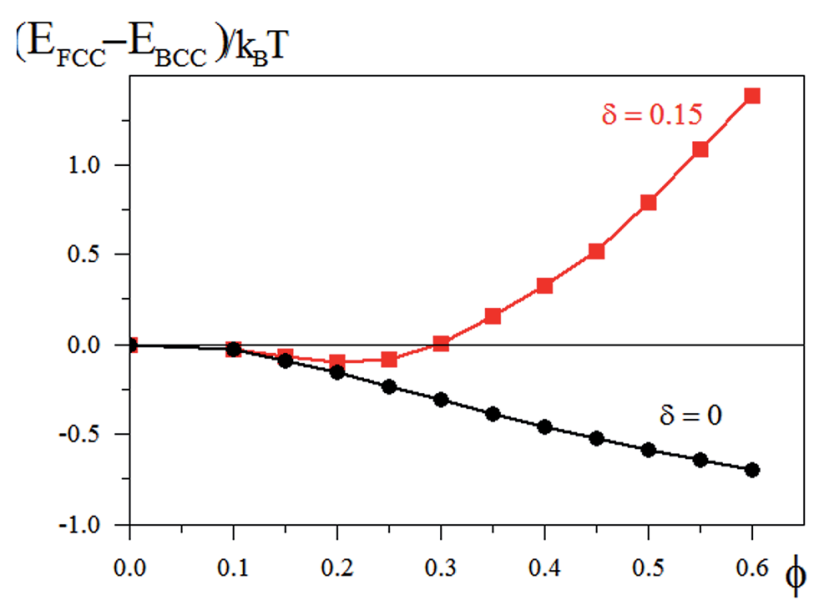

Fig. 1 Differences between the energies per particle of the FCC crystal and of the BCC crystal, at equilibrium, versus the system volume fraction, $\phi$, for particles of average radius $\langle a\rangle=8 \mathrm{~nm}$, Debye length $l_{D}=3 \mathrm{~nm}$, density of charge $=0.4 \mathrm{~nm}^{-2}$. Black circles are the exact calculation for the monodisperse case $(\delta=0)$; red squares are from the Metropolis Monte-Carlo method, 500000 random particle-exchanges for each of the 50 realizations of the random radius-distribution with polydispersity $\delta=0.15$, on the 2662 sites of the BCC crystal, or the 2916 sites of the FCC crystal. Positive values of the energy difference indicate that the BCC structure should be the stable phase at equilibrium. Then, polydispersity favors the BCC structure for the high volume fractions.

This is a sign of the correlated organization of the charges in the system, decreasing the energy of the BCC crystal more efficiently than for the FCC structure.

\section{Q2: how do the polydisperse particles distribute in a system with the BCC crystal phase coexisting with FCC crystal phase?}

A2: to understand why BCC becomes the stable phase when polydispersity is high enough, we consider the problem of the 2-phase system (coexistence): let us then suppose that the population of the polydisperse particles is arranged in part as a BCC lattice and in part as a FCC lattice, with proportions $\rho_{\mathrm{BCC}}$ and $\rho_{\mathrm{FCC}}=1-$ $\rho_{\text {BCC }}$, respectively.

To know the thermodynamic equilibrium of the system, we use a variant of the Gibbs ensemble Metropolis random exchanges of particles, introduced to study the thermodynamic evolution of different phases coexisting in a liquid. ${ }^{9}$ Particles are exchanged randomly between the phases according to the usual Metropolis Monte-Carlo scheme, keeping constant throughout the total number of particles, the total volume of the system and the temperature. In this method, the homogeneous coexisting phases are simulated in separated boxes without interfaces between the phases, and each box has periodic boundary conditions. Particle exchanges occur either inside a single phase, or from different phases.

To make the approach more efficient, we sampled the configuration space into a finite number of configurations, for example taking the 19 configurations 
corresponding to the coexistence of one cubic BCC crystal and one cubic FCC crystal, the total sum of the sites being between 3500 and 4500 (in order to compare systems of about the same total sizes). When thermodynamic equilibrium is reached in every configuration, the total energy, $E$, of the system is evaluated for each configuration. The program then scanned, little by little, the energy landscape of (a sampling of) the configuration space, and the approximate partition function $Z=\sum_{\text {conf }} \exp \left(-E / k_{\mathrm{B}} T\right)$ gives access to all thermodynamic quantities at equilibrium. The average value of the proportion of the BCC phase at equilibrium is estimated through:

$$
\left\langle\rho_{\mathrm{BCC}}\right\rangle=\frac{1}{Z} \sum_{\text {conf }} \rho_{\mathrm{BCC}} \mathrm{e}^{-E / k_{\mathrm{B}} T}
$$

and similarly for $\left\langle\rho_{\mathrm{FCC}}\right\rangle$.

Let us discuss the results for the value $\phi=0.3$ realizing the equality of the pure BCC and pure FCC energies, as seen in Fig. 1 . As could be expected, $\left\langle\rho_{\mathrm{BCC}}\right\rangle=$ $\left\langle\rho_{\mathrm{FCC}}\right\rangle=0.5$ is obtained in this case because of the choice $E_{\mathrm{BCC}}=E_{\mathrm{FCC}}$ for the pure phases. However, the actual value of the energy per particle for BCC and FCC crystals of the same size, is found to be $E=22.2 k_{\mathrm{B}} T$, significantly lower than the value for the single phase $E_{\mathrm{BCC}}=E_{\mathrm{FCC}}=23.0 k_{\mathrm{B}} T$. This means that the system put different particle distributions in the different phases. This occurrence is exemplified in Fig. 2 where the at-equilibrium radius distributions in the two phases are compared for the configuration corresponding to a BCC crystal of 2000 sites, and a FCC crystal of 2048 sites. The radius-distribution in the FCC phase has approximately a Gaussian shape with a polydispersity of $6.5 \%$, that is much smaller then the polydispersity (15\%) of the total distribution including all the

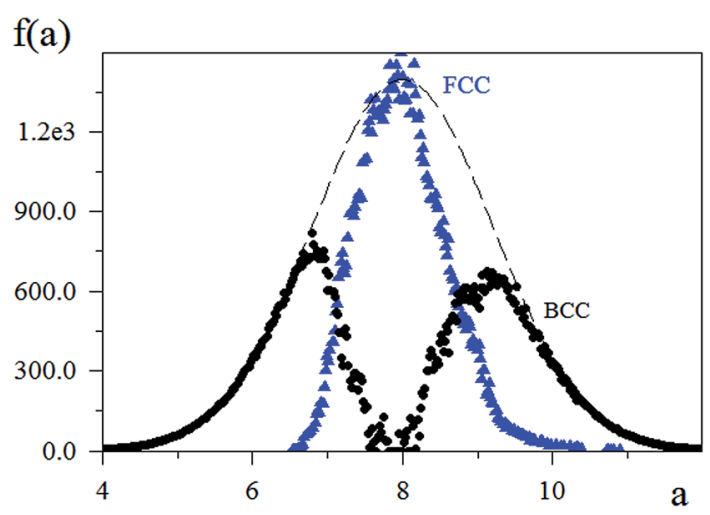

Fig. 2 At-equilibrium radius-distributions of the particles located in the phases of a system made of a FCC finite lattice of 2048 sites and of a BCC finite lattice of 2000 sites, both with periodic boundary conditions. The Gibbs Monte-Carlo scheme was applied with 300000 random particle exchanges and 50 random realizations of the total radiusdistribution (plotted as the dashed curve) with polydispersity $\delta=0.15$, for the system volume fraction $\phi=0.3$, all other parameters as listed in the caption of Fig. 1 . The FCC phase takes all the particles with radii close to the average value $\langle a\rangle$, letting the wings of the distribution be managed by the BCC lattice. The polydispersity of the population of particles making the FCC lattice is 0.065 , much smaller than the polydispersity of the total distribution, $\delta=0.15$. 
particles in the system. Consequently, both wings of the initial Gaussian distribution distribute in the BCC phase.

\section{Q3: why does the BCC crystal phase accept handling two modes of particle radius while FCC sticks to one mode?}

A3: the detailed management of the initial radius-distribution by the system results from the different abilities of the phases to accept particles of different radii (that is here, of different electric charges). Following this idea, one can define a parameter of tolerance as the maximum relative decrease of the system energy when changing the charges of a couple of particles, keeping the total charge unchanged. More precisely, let us define the tolerance, $\tau$, by the expression:

$$
\tau=\min \left\{-\frac{\Delta E}{U_{\mathrm{o}}}\right\}
$$

in which $\Delta E$ is the variation of the system energy when the charges of two particles are changed from the initial values $Z$ to: $2 Z$ and 0 , respectively, and $U_{\mathrm{o}}=Z^{2} \kappa l_{\mathrm{B}}$ is a reference energy for the system. The minimum value in (3) is taken over all the possible positions of the couple of particles with modified charge. This concept supposes that the system is able to detect the best (from the point of view of the energy) relative positions of the two concerned particles. Then the repulsive interactions are expected to be long-ranged - as the Yukawa potential is - even if the characteristic lengths are finite. For a regular lattice with only one sort of site, the value of $\tau$ is given by the expression:

$$
\tau=\frac{\mathrm{e}^{-\kappa r_{\mathrm{o}}}}{\kappa r_{\mathrm{o}}}
$$

where $r_{\mathrm{o}}$ is the value of the distance between the nearest neighbours.

For the BCC lattice of volume fraction $\phi$, one has $r_{\mathrm{o}} / a=(\pi \sqrt{27} / 3 \phi)^{1 / 3}$, and for the FCC lattice at the same volume fraction: $r_{\mathrm{o}} / a=(\pi \sqrt{32} / 3 \phi)^{1 / 3}$. Then, $\tau_{\mathrm{BCC}}>\tau_{\mathrm{FCC}}$ for all values of $\phi$ (for $l_{\mathrm{D}}=3 \mathrm{~nm}$ and $a=8 \mathrm{~nm}$ considered here: $\tau_{\mathrm{BCC}} / \tau_{\mathrm{FCC}}=1.3$, for $\left.\phi=0.3\right)$. This indicates that the FCC crystal phase is less tolerant than BCC, or, equivalently, that changing the charges of two particles as follows: $Z \rightarrow 2 Z$, and $Z \rightarrow 0$, results in a larger decrease of the BCC lattice energy than in the FCC case. This is the reason why, from the point of view of the energy, the system prefers to fill the FCC lattice with particles of almost the same charges, and to arrange the rest of the particles in the BCC lattice.

As a conclusion, we propose here a 'tolerance rule' of the form: in the case of coexistence of several phases, the less tolerant phase prevails over all the other phases in the distribution of the radii.

\section{Q4: what information can the method give about experiments of compression of a system of charged polydisperse particles?}

A4: as explained in the Introduction, we recently explored the colloidal crystallization of polydisperse Ludox HS silica nanoparticles dispersed in water through 
near-equilibrium dialysis to achieve very slow compression of the polydisperse suspension. ${ }^{1}$ We demonstrated that, in charge-stabilized colloidal dispersions at intermediate compressions, the system exhibits two crystalline phases, namely: a $\mathrm{BCC}$ phase and a Laves $\mathrm{AB}_{2}$ phase, in coexistence with the liquid. Moreover, the FCC phase is missing. The Laves $\mathrm{AB}_{2}$ crystalline system is known to be relevant for the opal structure, ${ }^{10}$ and more generally for packing of bidisperse particles ${ }^{11}$ with a radius ratio of $\sqrt{2 / 3} \simeq 0.82$. The tolerance of this phase, as defined in (3), is such that: if $\tau_{\mathrm{AB}_{2}} / \tau_{\mathrm{BCC}}=3.55$, in the experimental conditions, then the Laves lattice is much more tolerant than BCC to accepting uneven particles. Therefore, an argument similar to the one developed in Section $\mathrm{B}$, leads to the conclusion that the BCC phase should collect most of the particles close to the mode of the radiusdistribution, while the wings of the distribution should fill the $\mathrm{AB}_{2}$ phase.

To be more quantitative, the Gibbs ensemble Monte-Carlo method was used for the system made of a $\mathrm{BCC}$ phase in coexistence with an $\mathrm{AB}_{2}$ phase. As previously, the ensemble average of each crystalline phase proportion is evaluated after sampling the configuration space. This results in the ensemble-average values: $\left\langle\rho_{\mathrm{BCC}}\right\rangle=0.52 ;\left\langle\rho_{\mathrm{AB}_{2}}\right\rangle=0.48$ corresponding to the respective volume proportions of the phases at equilibrium. Then, considering a system close to these values, that is: a BCC crystal of $9 \times 9 \times 9$ unit cells, and a Laves $\mathrm{AB}_{2}$ crystal of $5 \times 5 \times 5$ unit cells, the resulting respective radius-distributions are plotted in Fig. 3 .

Here too, we find that the less tolerant phase (the BCC phase in this case) collects most of the particles with radii close to the average radius. The final polydispersity of the BCC phase particles is $7.5 \%$ in this example, emphasizing the role of the repulsive interactions in the process, since this polydispersity significantly exceeds the terminal value $5.7 \%$ of the polydispersity of the crystal phase made of hard sphere colloidal particles precipitating from a fluid phase at equilibrium. ${ }^{12,13}$ Moreover, the $\mathrm{AB}_{2}$ phase is pretty well filled with particles with a particle-radius ratio of about 0.8 , the bigger particles being located in the octahedral sites, and the smaller ones in the tetrahedral sites.

This suggests a simple "rule of thumb" for the respective proportions of the two phases: suppose that the radii of the particles filling the less tolerant phase are distributed in the Gaussian shape with polydispersity smaller than the overall polydispersity $\delta$, and almost all the particles of radius $\langle a\rangle$ belong to this phase. Calculating the two modes of the remaining radius-distribution of the particles in the high-tolerant phase is then a simple exercise. If we constrain the ratio between the two radius modes to be $\sqrt{2 / 3}$ for the system to efficiently fit the $\mathrm{AB}_{2}$ phase, $\uparrow$ then one finds the relation:

$$
\rho_{\mathrm{B}} \simeq \frac{\delta^{*}}{\delta}
$$

with $\rho_{\mathrm{B}}$ the proportion of the less-tolerant phase at equilibrium, and $\delta^{\text {出 }}=5-\sqrt{24} \simeq 10 \%$. In (5), we approximated: $2 \ln \rho_{\mathrm{B}} /\left(1-1 / \rho_{\mathrm{B}}^{2}\right) \simeq \rho_{\mathrm{B}}$.

Interpretation of eqn (5) is as follows: when $\delta<\delta^{*}$ only the less-tolerant phase (here: BCC) can exist; when $\delta>\delta^{*}$, the proportion of the BCC phase is a decreasing

\footnotetext{
$\dagger$ In principle, one should consider the ratio of effective radii based on the energy density. This does not change eqn (5) but the value of the limit of polydispersity $\delta^{*}$ which generally depends on the system volume fraction.
} 


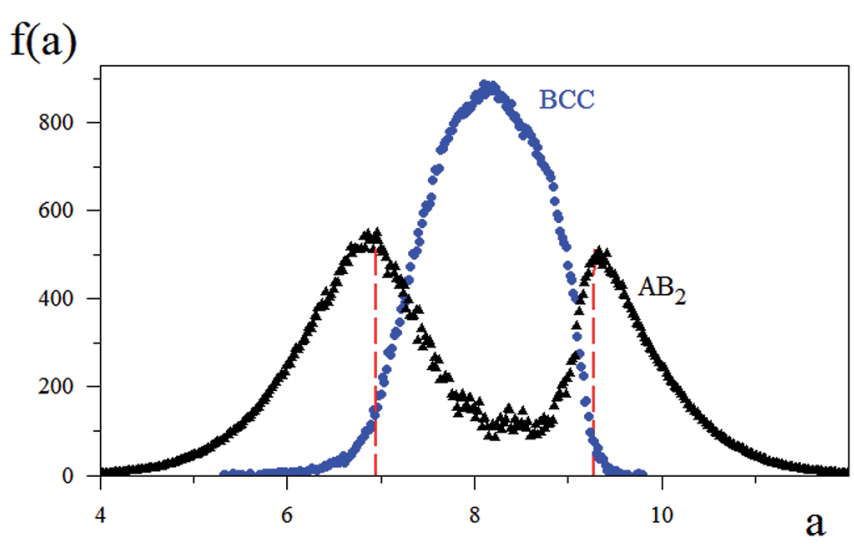

Fig. 3 At-equilibrium radius-distributions of the particles located in the phases of a system made of a BCC crystal of 1458 sites and of a Laves $A_{2}$ crystal of 1500 sites, both with periodic boundary conditions. The Gibbs Monte-Carlo scheme was applied with 300000 random particle exchanges and 50 random realizations of the total Gaussian radius-distribution with $\langle a\rangle=8 \mathrm{~nm}$, polydispersity $\delta=0.15$, for the system volume fraction $\phi=0.3$, all other parameters were as listed in the caption of Fig. 1 . The BCC phase takes most of the particles with radii close to the average value $\langle a\rangle$, letting the wings of the distribution be managed by the $\mathrm{AB}_{2}$ lattice. The polydispersity of the population of particles making the $\mathrm{BCC}$ lattice is $7.5 \%$ compared with the polydispersity of the total distribution, $\delta=15 \%$. The two modes of the $A B_{2}$ radius-distribution are the vertical red segments, respectively at $6.9 \mathrm{~nm}$ for the tetrahedral sites, and at $9.3 \mathrm{~nm}$ for the octahedral sites. The ratio between these two values is 0.74 , close to the value 0.82 for the $A B_{2}$ packing of hard spheres. Compared with Fig. 2, one can easily understand that the system prefers the present configuration for which all the modes are nearly at the correct place (unlike the BCC phase in Fig. 2, which had to be filled with a bi-disperse distribution).

function of the polydispersity, $\delta$, of the system, that is: polydispersity favors the search for more tolerant phases.

It is worth noting that the appearance of the Laves phase in this context can be related to a work on bidisperse particles. ${ }^{14}$ Indeed, such complex crystal structures were found to be spontaneously stabilized in the case of mixtures of spherical particles with two different diameters, interacting via simple repulsive potentials. This was shown by numerical simulations using the thermodynamic integration technique. However, our case is a priori much more surprising since there is not, at the beginning, any 'magic' radii ratio.

\section{Q5: how long-ranged should the interaction be between the particles?}

A5: the physical parameters that we have considered up to now are rather typical of short-range interactions, namely: an exponential decrease in (1) with a characteristic length $l_{\mathrm{D}}=3 \mathrm{~nm}$, much smaller than the nearest-neighbor distances, which are of the order $20 \mathrm{~nm}$. Then, it is natural to ask the question whether only the nearest-neighbors are important or if the farther neighbors play a role too.

A quick answer is given considering the monodisperse case for the BCC or FCC lattices. In these cases, one knows the analytical formula for the energy per 


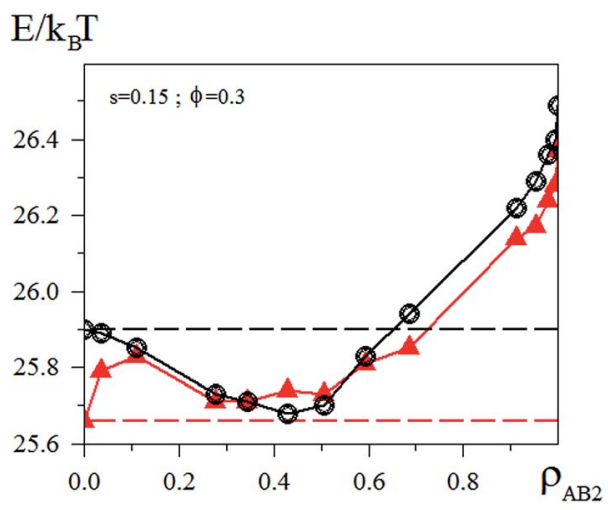

Fig. 4 Energies at equilibrium per particle of the system of the $\mathrm{BCC}$ crystal phase in the proportion $1-\rho_{\mathrm{AB}_{2}}$ coexisting with a Laves $\mathrm{AB}_{2}$ crystal phase in the proportion $\rho_{\mathrm{AB}_{2}}$. Equilibrium is reached after 300000 random particle exchanges and 50 random realizations of the Gaussian radius-distribution with polydispersity $\delta=0.15$, for the system volume fraction $\phi=0.3$ and total number of sites between 2500 and 3500, all other parameters as listed in the caption of Fig. 1. The open circles are the total Yukawa interaction, while the red triangles are the Yukawa interaction truncated at the nearestneighbors (the respective black and red continuous line are guides for the eyes). The black (respectively red) dashed line is the energy of the pure BCC phase considering all the neighbors (respectively only the first-neighbors). The statistical error bars are of the order $0.1 k_{\mathrm{B}} T$. When all the neighbors are included, the system energy reaches the minimum value for $\rho_{\mathrm{AB}_{2}} \simeq 0.42$ (to be compared to the average value $\left\langle\rho_{\mathrm{AB}_{2}}\right\rangle \simeq 0.48$ found in Section 6 using the partition function). On the other hand, all the results including only the nearestneighbors are found to be above the energy of the pure BCC lattice, indicating that the system should not consider the $A B_{2}$ phase if the Yukawa interaction was truncated. As a matter of fact, the difference between the nearest-neighbors/all-neighbors is essentially important only for the less tolerant crystalline structure in this case (i.e. the BCC phase).

particle as a series in the order of the neighbors, then it is easy to compare the energy when only the nearest-neighbors are considered. For the same parameters as in the caption of Fig. 1 and $\phi=0.3$, only the first-neighbors give the results: $E_{\mathrm{BCC}}^{(1)} / k_{\mathrm{B}} T=24.98<E_{\mathrm{FCC}}^{(1)} / k_{\mathrm{B}} T=29.79$ which seems to show that the BCC crystal is more stable than the FCC crystal. But including the second-neighbors turns round that conclusion, as: $E_{\mathrm{BCC}}^{(2)} / k_{\mathrm{B}} T=30.47>E_{\mathrm{FCC}}^{(2)} / k_{\mathrm{B}} T=30.32$. Taking into account all the neighbors confirms the latter conclusion: $E_{\mathrm{BCC}}^{(\infty)} / k_{\mathrm{B}} T=30.82>E_{\mathrm{FCC}}^{(\infty)} /$ $k_{\mathrm{B}} T=30.51$. Actually, this discussion was about the point $\delta=0 ; \phi=0.3 ;\left(E_{\mathrm{FCC}}-\right.$ $\left.E_{\mathrm{BCC}}\right) / k_{\mathrm{B}} T=-0.31$ of Fig. 1 . Then, even if $l_{\mathrm{D}} / r_{\mathrm{o}} \ll 1$, one must consider more than only the nearest-neighbors to get the correct conclusion about the stability of the crystals.

To check the relevance of this 'long-range versus short-range' question in the case of the coexistence of the $\mathrm{BCC}$ and $\mathrm{AB}_{2}$ phases of polydisperse particles, we cut the Yukawa potential (1) right after the first nearest-neighbors (three kinds of sites are to be considered: one for the BCC lattice, the tetrahedral site and the octahedral site of the $\mathrm{AB}_{2}$ lattice). Then, the second- and farther neighbors of a given particle do not interact with it any more. The main results are conveniently visualized in Fig. 4, after plotting the energies per particle of the system in phase coexistence versus the composition of the system (characterized by: $\rho_{\mathrm{BCC}}=1-$ $\rho_{\mathrm{AB}_{2}}$ and $\rho_{\mathrm{AB}_{2}}$ as the respective volume proportions). 
The conclusion is that the number of neighbors considered is relevant in the energy profile versus the composition of the system. In the example in Fig. 4, the system is probably a pure BCC crystal phase if the Yukawa interaction is cut at the first-neighbors, while coexistence of the $\mathrm{BCC}$ and $\mathrm{AB}_{2}$ phases is clear when all the neighbors are taken into account.

\section{Conclusions - what did you learn from the method?}

In the case of the fractionated crystallization, the Gibbs ensemble Monte-Carlo method does not predict the nature of the phases at equilibrium. However, it can provide essential help for a quantitative description of the crystals in coexistence (such as: the respective volumes of the phases, the particle size-distributions in each phase, etc.) as well as the fundamental mechanisms leading to the final composition at equilibrium, within the framework of the DLVO theory. In the two cases presented here (the FCC $+\mathrm{BCC}$ and the $\mathrm{BCC}+\mathrm{AB}_{2}$ systems), spontaneous self-assembly processes sort out particles from a broad polydisperse population and build different sets of colloidal crystals with structures that are adapted to a best use of this population. One process selects the most popular particles to build a first set of crystals (the less tolerant crystal) and "burns" a hole in the original population of particle sizes. The residual particle population then resembles a bi-disperse mixture, and a second process makes use of the remaining sub-populations on either side of the "hole" to construct other crystals (more tolerant). This process of sieving is probably general and leads naturally to a sequence of fractionated colloid crystallization governed by the tolerance rule. On the basis of what we know about binary nanoparticle systems, ${ }^{11,15}$ we can expect still more complex superlattices to be built spontaneously that way for still larger polydispersities.

This description of the mechanisms is close to the idea by Pusey ${ }^{\mathbf{1 6}}$ in a different context - an idea which has been validated much later through numerical simulation $\mathrm{s}^{\mathbf{1 7}}$ and theoretical developments ${ }^{\mathbf{1 3}}$ - that suspensions of highly polydisperse hard spheres might crystallize by splitting the broad overall distribution into a number of narrower distributions, each of which being accommodated within a single crystalline phase.

We shall close this presentation with three discussions about the limitations of the numerical method.

- The method does not consider the real kinetics of the mechanisms, then reliability of the method for large volume fractions may be questionable since movements of the particles can be hindered. However, for systems of spherical particles, repulsive interactions act as lubrication between the particles and generate ergodicity. In other words, local rearrangement of the particles (related to the system capability to reach thermodynamic equilibrium) is facilitated by the repulsive interactions, and more precisely by the value of the energy at contact. This is not the case in the opposite situation of attractive interactions, resulting generally in non-equilibrium patterns. ${ }^{18}$

- In principle, the method cannot predict the phases at equilibrium, since it supposes to have a priori information - or to guess - about the phases in coexistence. However, one should note that if large unit crystal cells are being formed 
in the system, no numerical kinetic method (e.g. molecular dynamics) can nowadays predict the phases, due to essential computing limitations.

- A last but fundamental question is the role of the liquid phase in coexistence with the crystals. In principle, the same Gibbs ensemble Monte-Carlo method for a system of two crystal phases plus a liquid phase can be used if a fast-computing free energy method is at hand for the liquid phase. The optimized random-phase approximation (ORPA) is a good candidate for a systematic study. This approach could help to solve the similar problem with large polydispersities. Indeed, in this case, the wings of the charge-distribution function are probably too wide to be incorporated in the only Laves $\mathrm{AB}_{2}$ phase, and the system should choose the liquid phase as a reservoir of unfitting particles (too large or too small), expressing the high tolerance of the liquid phase. However, experimental data for such systems with large polydispersity are scarce, and it is not clear whether such a system will choose intensive use of the liquid phase (i.e. melting the phases) or if it will find another crystalline structure with a still larger crystal unit cell.

\section{Acknowledgements}

Part of this work was realized in the framework of the GDR AMC2 "Approches Multi-Physiques pour les Colloïdes Concentrés”, CNRS. We thank Christophe Labbez and Guillaume Bareigts for interesting comments about the Monte-Carlo numerical method used in this work.

\section{References}

1 B. Cabane, J. Li, F. Artzner, R. Botet, C. Labbez, G. Bareigts, M. Sztucki and L. Goehring, Hiding in plain view: Colloidal self-assembly from polydisperse populations, 2015, ArXiv: 1511.02493v1

2 F. Laves, Crystal Structure and Atomic Size, in Theory of Alloy Phases, The American Society of Metals, Cleveland, OH, 1956, pp. 124-198.

3 A. Z. Panagiotopoulos, in Observation, prediction and simulation of phase transitions in complex fluids, ed. M. Baus, L. R. Rull and J. P. Ryckaert, Kluwer Academic Publishers, Dordrecht, The Netherlands, NATO ASI Series C, 1995, vol. 460, pp. 463-501.

4 E. Zaccarelli, S. M. Liddle and W. C. K. Poon, Soft Matter, 2015, 11, 324-330.

5 E. J. Verwey and J. T. G. Overbeek, Theory of the Stability of Lyophobic Colloids, Elsevier, Amsterdam, The Netherlands, 1948.

6 I. Sogami and N. Ise, J. Chem. Phys., 1984, 81, 6320-6332.

7 Ordering and Phase Transitions in Charged Colloids, ed. A. K. Arora and B. Tata, Wiley-VCH, New York, 1996.

8 M. O. Robbins, K. Kremer and G. S. Grest, J. Chem. Phys., 1988, 88, 3286-3312.

9 The Monte-Carlo Method in Condensed Matter Physics, Topics in Applied Physics, ed. K. Binder, Springer-Verlag Berlin Heidelberg, New York, 1992, vol. 71.

10 M. J. Murray and J. V. Sanders, Philos. Mag. A, 1980, 42, 721-740.

11 Z. Chen and S. O'Brien, ACS Nano, 2008, 2, 1219-1229.

12 D. A. Kofke and P. G. Bolhuis, Phys. Rev. E: Stat. Phys., Plasmas, Fluids, Relat. Interdiscip. Top., 1999, 59, 618-622.

13 M. Fasolo and P. Sollich, Phys. Rev. Lett., 2003, 91, 068301. 
14 M. D. Eldridge, P. A. Madden and D. Frenkel, Nature, 1993, 365, 35-37.

15 L. Filion and M. Dijkstra, Phys. Rev. E: Stat., Nonlinear, Soft Matter Phys., 2009, 79, 046714.

16 P. N. Pusey, J. Phys., 1987, 48, 709-712.

17 P. Bartlett, J. Chem. Phys., 1998, 109, 10970-10975.

18 G. Foffi, C. de Michele, F. Sciortino and P. Tartaglia, J. Chem. Phys., 2005, 122, 224903. 